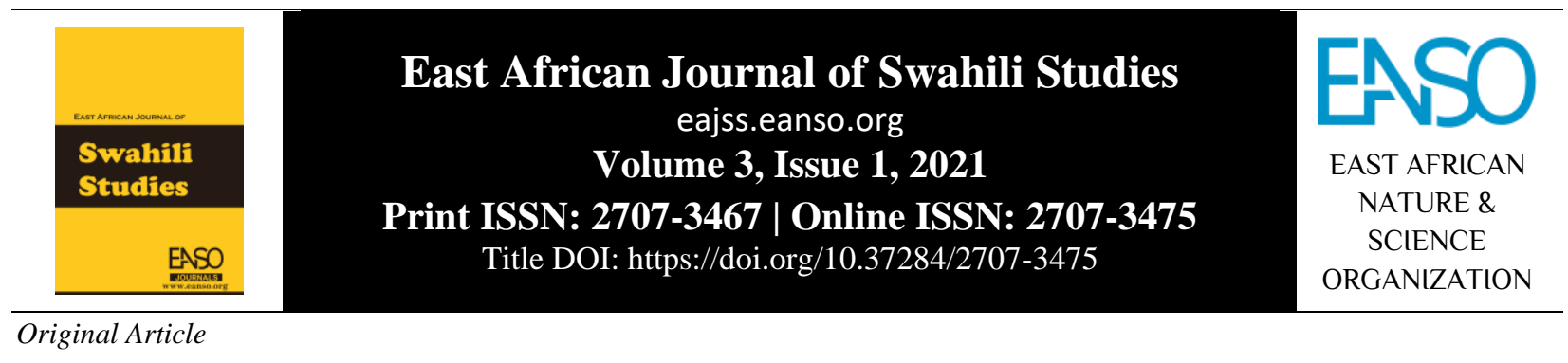

\title{
Ni Mvița ni Mvita?: Uchanganuzi wa Usuli wa Toponimi za Mithaa ya Mambasa, Kenya
}

\author{
Mohamed Karama \\ ${ }^{1}$ Chuo Kikuu cha Kabianga, S.L.P 2030 - 20200, Kericho, Kenya. \\ *Barua pepe ya mawasiliano: mkarama@kabianga.ac.ke
}

DOI ya Nakala: https://doi.org/10.37284/eajss.3.1.368

\section{Tarehe ya IKISIRI \\ Uchapishaji:}

Miji yothe ulimwenguni imepawa majina ili kuitambulisha. Majina haya ni hazina

27 Juli 2021 ya mapisi na utamaduni kuwahusu wakaaji wa miji hiyo. Namna inavoitwa, fasiri na fasili za majina hayo husema mengi kuhusu maana na nyusuli zake. Makala haya Istilahi Muhimu: yamechunguza toponimi ya baadhi ya mithaa ya mji wa Mambasa, Kenya. Madhumuni yalikuwa ni kuchanganuwa nyusuli za majina kwa kubainisha tafauti Mambasa, za kimatamshi ya majina hayo, kuthibitisha kuwa tafauti hizi zaleta tafauti ya maana Mvita, ya majina, na kwamba toponimi za mit ${ }^{\text {haa }}$ hiyo imeelemeya kwelezeya maumbile Toponimi, ya mazingira ya mahali hapo jambo linaloshuhudiwa katika jamii nyengine za Kibantu, Kenya. Tulitumiya Taratibu za Ulinganisho na Toponimi Kina ili Isimu, kuchanganuliya data yetu. Data yetu tuliipata kwa kuwauliza wazee wa miyaka 50 Swahili, na zaidi ambao wana maelezo simulizi kuhusu fasili za majina hayo na data ya upili. Mombasa. Kwa kulinganisha na lugha za jamii zinazokaribiyana za Kimvita, Kimijikenra, Kipokomo na lugha za Kibantu kutoka bara Kenya tuliweza kung'amuwa kuwa majina haya ya Mambasa yanashabihiyana na yale ya lugha nyengine hivo kutupa ufunuwo mpya kuhusu maana za majina hayo. Tumepata kuwa utamkaji wa majina unaleta tafauti ya maana na hivo usuli wao piya ni tafauti na ilivozoweleka. Tumepata kuwa majina ya kale yaliitwa kutegemeya maumbile ya mazingira na majina ya mithaa ya kisasa yameelemeya zaidi vitu au majengo yaliyoko hapo. Kupitiya makala haya tunaamini tumetowa fasili mpya kuhusu mithaa hii na hivo kuwapa fikira nyengine wataalamu wa Chimbo, Utalii na Isimu kuhusu majina haya na historiya inayobebwa na majina haya.

\section{APA CITATION}

Karama, M. (2021). Ni Mvita ni Mvita?: Uchanganuzi wa Usuli wa Toponimi za Mithaa ya Mambasa, Kenya. East African Journal of Swahili Studies, 3(1), 78-90. https://doi.org/10.37284/eajss.3.1.368.

\section{CHICAGO CITATION}

Karama, Mohamed. 2021. "Ni Mvita ni Mvita?: Uchanganuzi wa Usuli wa Toponimi za Mithaa ya Mambasa, Kenya”. East African Journal of Swahili Studies 3 (1), 78-90. https://doi.org/10.37284/eajss.3.1.368.

78 This work is licensed under a Creative Commons Attribution 4.0 International License. 


\section{HARVARD CITATION}

Karama, M. (2021) "Ni Mvita ni Mvita?: Uchanganuzi wa Usuli wa Toponimi za Mithaa ya Mambasa, Kenya”, East African Journal of Swahili Studies, 3(1), pp. 78-90. doi: 10.37284/eajss.3.1.368.

\section{IEEE CITATION}

M. Karama, "Ni Mvita ni Mvita?: Uchanganuzi wa Usuli wa Toponimi za Mithaa ya Mambasa, Kenya", EAJSS, vol 3, no. 1, pp. 78-90, Jul. 2021.

\section{MLA CITATION}

Karama, Mohamed. "Ni Mvita ni Mvita?: Uchanganuzi wa Usuli wa Toponimi za Mithaa ya Mambasa, Kenya". East African Journal of Swahili Studies, Vol. 3, no. 1, Jul. 2021, pp. 78-90, doi:10.37284/eajss.3.1.368.

\section{UTANGULIZI}

Tangu Shakespeare (ca. K17) mpaka Wamitila (1999), Mazrui (2012), hadi Owino (2019), na juzi Mutua (2021) wameuliza swali 'Linani jina?' na kila mmoja ameeleza fasili yake kuhusu mifumo ya kwita majina ya wat ${ }^{\mathrm{h}} \mathrm{u}$, mahali au $\mathrm{k}^{\mathrm{h}} \mathrm{itu}$. Tukiinukiya tulisomeshwa skuli na tulisoma magazetini majina ya miji na nchi: Peking, Bombay, Ivory Coast na baada ya miyaka michache miji hii sasa yaitwa/yaandikwa: Beijing, Mumbai, na Côte d'Ivoire kimtawaliya. Huku Uswahilini-Kenya, imepita miyaka miya moja tangu wanathi wa Mambasa, wakinukuliwa katika Stigand (1915: ix), waliposhangazwa na maelezo ya maana ya jina la mji wao 'Mvita' kwamba ni 'mji wa vita' 'kisiwa kilichojificha', 'mji wa ncha'. Tukiyengezeya, nyanjani, kuna wanaosema usuli wa jina 'Mambasa' ni 'min bas', 'mambo sasa', na 'mwambadze'. Ni dhahiri majina yana umuhimu wa kumtambulisha $\mathrm{m}^{\mathrm{t}} \mathrm{u}$ kule atokako, lakini piya utamkaji, kama tutakavothibitisha, wa jina hilo una mchango katika utambulisho huwo. Taratibu ya kuchanganuwa etimolojiya ya toponimi inafafanuwa kuwa majina ya mahali hutrokana na mazingira au matukiyo muhimu yanayonasibishwa na mahali hapo. Katika makala haya, tutatumiya taratibu hii kutatuwa mdangano na kwangaziya mwelekeyo wa nyusuli za majina ya kale ya mithaa ya Mambasa.

Kupitiya maandiko tumejuwa Mambasa ilikuwa ikiitwa $\mathrm{K}^{\mathrm{h}}$ ongoweya, Mvita, Jimbo, Gongwa, Nyali $\mathrm{K}^{\mathrm{h}} \mathrm{uu}$, Nuhasi, Mji wa Kale (Abdulaziz, 1979:146). Naye Muyaka bin Haji katika Abdulaziz ametupa majina tafauti tafauti ya mji huu kiushairi bila kugusiya nyusuli za majina hayo. Maelezo ya Abdilatif Abdalla (mazungumzo naye kwa WhatsApp, April 25, 2019) yanaeleza baadhi ya nyusuli za majina ya Mambasa na Mvita kulingana na maandiko yaliyoko, kwa mfano, 'nabasa' (kusema hadharani) na 'Manpasa' kulingana na
James Kirkman, Shihabudin Chiraghdin mtawaliya. Tunakubaliyana naye kuhusu namna ya kwita majina lakini kuhusu nyusuli maelezo yake hayakubaliyani na utaratibu wetu wa utafiti kama tutakavoonesha. Kazi ya Owino (2019) imeeleza kuhusu athari na tahadhari za kisheriya zilizoko katika kwita majina haswa ya wat ${ }^{\mathrm{h}} \mathrm{u}$ na bidhaa. Mazrui (2012) na Mutua (2021) wameangaziya siyasa iliyomo katika jina la mahali lakini ya Mutua imefanana na yetu kwa kwangaziya maswala ya toponimi ya jina 'Kenya' kiutamkaji wake; sisi hatutomili katika siyasa. Wamitila (1999) ameeleza fasili ya jina 'mvita' kwa mnasaba wa kazi za kifasihi na ametowa etimolojiya ya jina hili kiuhusika na wala si jina la mahali. Kazi ya Makoti (2007) imeeleza kuhusu anthroponimi kulingana na Wadigo na ikatufunuliya namna etimolojiya ya majina ya $w^{t^{h}} \mathrm{u}$ ya Kidigo yalivo na umuhimu katika utamadnuni na mapisi yao. Kazi ya odonimi ya Wanjiru na Matsubara (2017) imetufunuliya kuhusu uhifadhi wa historiya ya nchi na umuhimu wa historiya hiyo katika kujenga utaifa kutrokana na kwita majina ya barabara muhimu katika mji, haswa miji mikuu. Kazi ya Nzasu na Odongo (2014), kitabu cha kiyada cha Darasa la 4 katika skuli za msingi, kimegusiya, kwa muhtasari sana, pengine kwa kiwango cha walengwa, mas'ala ya aina za wakaaji katika mji wa Mambasa, tamadnuni zao, sifa za kijiografiya za maeneo ya mji huu na maenreleo yaliyomo. Kazi hii haikutaja wala kutrowa historiya angalau fupi ya mji huu mkongwe au toponimi yake. Karama (2021) inayohusu toponimi ya 'Swahili' imetupa fikra ya kwangaliya majina haya kwa kurejeleya nadhari ya Kiafrika badala ya kuwona kwamba majina haya yametokana na kubanrikizwa kutoka nje ya utamadnuni wa Waswahili, chembelecho Nurse na Spear (1985). Fasili tulizopata nyanjani zimedhihirisha kuwa $m t^{\text {thaa }}$ au mji Mvita hutamkwa namna mbili: Mvita na Mvita. Uchanganuzi wetu umethibitisha kuwa pana tafauti ya utamkaji na hivo yanaathiri maelezo 
ya nyusuli za jina hili nriposana twauliza, 'Kunani na utamkaji jina?'

Natija ya makala haya miyongoni mwao ni kutowa maelezo mengine kuhusu maana ya majina ya mji huu ili kwonrowa mdangano uliyoko, mfano wa Sacleux (1939: 632), Stigand (khj.) na Kresse (2007: 64). Fauka ya hayo, matokeo ya utafiti wetu yatrawasaidiya wataalamu wa ElimuChimbo kufunukiwa na mapisi ya mahali wanapochimbuwa kama wanavopendekeza watafiti Nurse na Spear (1985) na Walsh (2003). Mbali ya kujuwa mapisi ya majina haya, jambo lililojitokeza katika utafiti wetu ni kuwa tafauti za kutamka majina haya nrizo zili(na)zosababisha tafauti za maana ya majina haya. Hivo basi, pana haja ya kuhifadhi matamshi ya wasemaji kindi kindaki wa lugha ya Kiswahili ambayo yaonekana yana mchango katika kuufahamu vizuri mji huu na mengineo kimapisi.

\section{UȚARAȚIBU WA UȚAFIȚI}

Tarațibu za Isimu Mapisi na za Toponimi zilitumiwa kuchanganuliya data yetu ambayo ni baadhi ya majina ya mithaa ya mji wa Mambasa. Tulitumiya Utaratibu wa Ulinganisho (Bynon, 1977; Antilla, 1989; Campbell, 1998) wa visawe katika lugha ya Kiswahili na lugha nyengine za Kibantu jirani ya Kiswahili ili ufanano wa visawe hivo uweze kutufunuliya ufanano wa maana ya majina hayo. Visawe hivi vilichanganuliwa katika hati finyu kifonetiki za Kiswahili asiliya jambo ambalo Antilla amesema ni gumu. Walakini, kwa faida ya uchanganuzi wetu, sauti hizi nrizo zilizotufahamisha uwamilifu wake na hatuwa hii ni jambo wamelikubali Antilla na Campbell.

Timbo muhimu ya kupata asili ya maneno katika Isimu Mapisi huwa ni kwenye nyaraka za zamani ambazo hutwonesha namna lugha ilivokuwa ikitumiwa na wenyeji wakati wa nyuma (Bynon, 1977). Walakini, Kiswahili ni kati ya lugha ambazo hazikuwa na hati zake wala utamadnuni wake haukuhifadhiwa kwa uwandishi bali mengi ya maswala mbalimbali ya kijamii yalihifadhiwa nyoyoni mwa wasemaji wake na kupokezwa kizazi baadra ya kizazi kwa mdomo. Jambo la maandishi, kwanza kwa hati za Kiyarabu na baadaye kwa Kilatini, ni mambo ya hivi karibuni. Kwa sababu hiyo, wakati wa utafiti wetu, tulikwenra kwa wazee maarufu kwa mapisi ya Waswahili wa miyaka 50 na zaidi haswa ambao hawakuathiriwa sana na Kisanifu. Sampuli hii ya wahojiwa ilitufaa sisi kwa sababu ya kupata matamshi na fasili ya kisimulizi kuhusu majina haya. Data ya upili piya ilitumika ili kwengezeya lililokosekana kutoka kwa wasailiwa na piya kuchuja tulichopawa na wasailiwa. Uchanganuzi wa data ni wa kisinkroniya na uwasilishaji wake ni wa kimaelezo. Tuliyangaliya namna visawe va lugha hizi vinavofanana na kutafautiyana bila ya kwangaziya mabadiliko ya mpito wa wakati mpaka yakafikiya hali yalivo sasa. Mengi ya yanayoelezewa katika makala haya ni ya kidhahanifu lakini nrivo ulivo uchunguzi wa kisinkroniya - hutizama haliya ya lugha inavotumika katika jamii na kwiyelezeya huku ikirejeleya maandiko kupunguza udhahanifu huu (Bynon, 1977). Katika makala haya Kiswahili asiliya inawakilisha matamshi ya Kiswahili lahaja na Kisanifu kimetumika kuwakilisha Kiswahili Wastani. Tunapotaja lugha nyengine za Kibantu tumetumiya jina la jumla bila kutafautisha lahaja zilizomo nrani yazo.

Utaratibu wa toponimi uliyofafanuliwa na Tent (2015) unaonesha kuna njiya mbili za kufanya utafiti wa kitoponimi. Kuna wa kina ambao hushughulikiya majina asili zake kietimolojiya, kwa kufuwata misingi ya kiisimu, kinyaraka, au kifasiri. Njiya ya pili ni kwangaliya kwa ujumla majina yothe ya mji huwo na kuhitimisha kitakwimu kutokana na mifumo ya kwita majina hayo katika jamii husika. Katika makala haya tulitumiya njiya ya kwanza ambayo hwangaziya usuli kutokana na othografiya (namna lilivoandikwa [kwa mintarafu yetu kusemwa]), kimofolojiya, na kisemantiki kama alivofanya Helander (h.t.). Rose-Redwood na wenzake (2010) wameonesha kwamba utafiti wa toponimi hauna budi kuvuka mipaka ya etimolojiya na kwangaziya mas'ala ya siyasa na hisiya kuhusu majina ya mahali. Sisi tulijikita katika etimolojiya ya kimaumbile ya baadhi ya mithaa ya Mambasa ambayo nriyo yenye kuleta mdangano wa nyusuli zake. Tumechaguwa baadhi ya mit ${ }^{\text {haa }}$ kwa sababu ya ukale wake na piya uwafiki wa toponimi hizo katika misingi ya utafiti wetu na kutowa fikra ya utafiti kwa mithaa iliyosaliya.

Madhumuni yetu yalikuwa ni: mosi, kuthibitisha kuwa majina haya ya kale yanaelezeya zaidi vitu va kimaumbile. Pili, kuchanganuwa kiisimu-mapisi (mlinganisho wa fonolojiya, mofolojiya, na 
semantiki ya majina hayo) ili kuvumbuwa kuwa majina haya yamechukuliwa kivengine kimaana na yanaregeya kule kule kwenye majina ya kimaumbile. Tatu, na mwisho, ni kubainisha kuwa majina haya yana mshikamano na toponimi za miji mengine iliyopakana na Mambasa hivo kuyakinisha kuwa lugha hizi za Kibantu (Kumbo la Sabaki) katika Mwambao wa Pwani zina ukuruba wa kitamaduni, kimapisi, na kilugha kama alivosema Nurse na Spear (1985) na Makoti (2007).

\section{MUKTADHA WA MJI WA MAMBASA}

Mambasa, nrivo ustahikivo kwitwa kama tutakavothibitisha katika makala haya, ulibuniwa karne nyingi kabla ya kuja wageni wowothe. Yaweza kuwa hiyo Periplus ilipomaanisha miji ya wat $^{\text {th}} \mathrm{u}$ weusi Mambasa ilikuwako tayari. Hata wasafiri wa Kiyarabu, Al Idris (K 12) na Ibn Batuta (K 14), walipozitembeleya sehemu hizi wameitaja miji ya Mambasa na mengineo kuwa imestawi (Abdulaziz, 1979). Mji wa Mambasa uko katika kisiwa kwenye ufuwo wa pwaa za Afrika ya

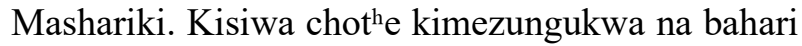
na kiko kwa nrani kimezibika na p $^{\text {hepo }}$ kali zinazopiga uso wa upwa huwo. Kaskazini kimepakana na bara la Kisauni, kusini ni bara la Likoni na Mtongwe, na magharibi kimepakana na bara la Changamwe na kwa mbali kidogo Rabai. Hupokeya $\mathrm{p}^{\mathrm{h}}$ epo za kusi zinazoleta mvuwa ya

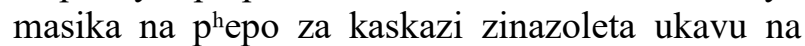
kiangazi kikali chenye joto jingi. Kuna na maleleji wakati kaskazi inapoikaribisha kusi na kipupwe wakați masika yanapoonroka na kukaribisha mchoo.

Neema hizi za kijiografiya nrizo zilizoifanya bandari ya Mambasa kuwa mahali pazuri pa kwegesha majahazi ya kutuwa kuleta mali kutoka nchi za nje na piya mahali pa kupita kwenra mbele ya safari za baharini. Kazi za baharini zilivutiya wat ${ }^{\text {h}} \mathrm{u}$ wengi wa kutoka nrani barani na piya nchi za ng'ambo: India, Indonesia, China, na zaidi bara Arabu. Mtangamano huu ulileta jumuiya ya wat ${ }^{\mathrm{th}} \mathrm{u}$ changamano na piya ulisababisha kila mmoja kupiganiya fungu la utawala wa mji huu. Wenyeji walijitawala kiukoo na wakijitambulisha kwa mahali watokako. Wageni Wareno, Waarabu, Majarumani (walitawala Tanganyika), Maingereza walikuja na walijitwaliya utawala kwa nguvu. Kubakurana huku kuutwaa utawala wa mji huu kulileta vita vikubwa baina ya Waarabu na Wareno, Waarabu na wenyeji, Maingereza na wenyeji.

Tangu zamani Mambasa kulikuwako na ukulima mambo ambayo Ibn Batuta hakuwacha kuyataja. Piya walitegemeya kuletewa chakula chao kutoka miji ya $\mathrm{Ph}^{\mathrm{h}} \mathrm{emba}$ na Nyika (sehemu ya ardhi nrani kidogo ya Mambasa). Nikikuliya mimi kulikuwa na vitaru Bonreni (ilipo New Peoples Hotel), Kikowani na Allidina vikipanrwa maboga kwa ajili ya kula na kuuza sokoni. Mashamba makubwa yalikuwako kuvuka kisiwa magharibi, kusini na kaskazini mwake hata sehemu hizo zikiitwa 'shamba'.

Leo Mambasa ni mji mkubwa ulopanra cheo na kuwa Mji Mkuu wa Pili nchini Kenya. Tena, una bandari kubwa ya Kilinrini na uwanja wa nrege wa kimataifa. Mashamba mengi yashafekwa na majumba makubwa kuchukuwa mahala pake na mji kupangika kisasa hata kukawa na mit ${ }^{\text {haa }}$ yaitwa Majengo Mapya, Magorofani huko huko kulikokuwa kukiitwa 'shamba'.

\section{MAJINA YA MITh'AA YA MAMBASA}

Sacleux (1939:604-5) ameorodhesha mithaa ya Mambasa na ameigawa kulingana na ukuruba na ngome ya Fort Jesus, ukale, usasa, na umbali wake na $\mathrm{m}^{\text {thaa }}$ wa zamani wa Mambasa yaani Mji wa Kale. Ametaja Ngomeni Fort Jesus (Kavani); mit ${ }^{\text {haa }}$ ya Mji wa Kale: Mkunguni, Msalani, Kilifi, Bondeni, Kikoani, Karoleni (Kaloleni); mithaa ya hivi karibuni: Makadara (Mt ${ }^{\mathrm{h}}$ aa wa waBulushi), Shehejundani; na mithaa ya kanro: Kilindini, Makupa, Mshimanzi, Baraki, Pwani wa Kisauni, Kizingo.

Orodha hii twaiyengeza, kwa faida ya data ya makala yetu, Baghani, Ingilani, Ganjoni, Liwat ${ }^{\mathrm{h}}$ oni, Ziwani, Kiziwi yothe ya kisiwani. Halafu kuna Nyali, $\mathrm{K}^{\mathrm{h}}$ ongoweya, Mishomoroni, Miyuchi, Bombolulu, Bumburi (siku hizi Bamburi), Utange, Maunguja. Upanre wa magharibi kuna Changamwe, Bomu, Mikinrani, Mik(g)adini, Jomvu $\mathrm{K}^{\mathrm{h}} \mathrm{uu}$, Jit ${ }^{\text {h}}$ oni. Kusini kuna Likoni, Mt ${ }^{\text {h}}$ ongwe. Mwisho kabisa ni mith aa ya hivi karibuni Kerekeța, Peleleza, Guraya, na Majengo (King'orani, Mferejini, Chura, Ropa, Sidiriya, Kipera (Floringi)). Tumeengeza mithaa hii kimakusudi ili wakati tunapochanganuwa 
aina za toponimi tuweze kufahamu kivipi mithaa ya kizamani na ya kisasa iliitwa hivo.

\section{Aina za toponimi ya Mithaa ya Mambasa}

Kulingana na Tent na Slatyer (2009) akinukuliwa na Tent (2015:73) anasema toponimi ni kumbukumbu za kutweleza sisi ni kina nani, lini tulikuja, na ni hazina $\mathrm{k}^{\mathrm{h}} \mathrm{uu}$ ya habari za historiya ya mahali pale. Katika taratibu hii ya utafiti wa toponimi, Tent amebayanisha aina kadhaa za toponimi na nriyo tuliyotumiya kwelezeya toponimi ya mithaa ya Mambasa.

1 Toponimi ya Mnasaba: ni ile inayoashiriya mnasaba wa mahali hapo na muktadha wake au

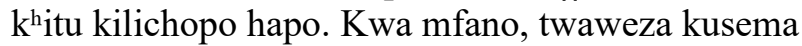
Lango la Papa, Madhubaha, Lighthouse (Mama Ngina) inanasibishwa na muktadha wa mahali hapo. Kwa mfano, Madhubaha, neno la Kiyarabu kwa maana ya 'kichinjiyo', karibu na Allidina School, kaskazini mwa kisiwa huwa pana sherehe ya kuchinja hapo kila mwaka wakati wa Mwaka Mpya wa Waswahili.

2 Toponimi ya Matukiyo: ni inayohifadhi tukiyo, tenro, sherehe fulani, au tarehe ya jambo lililotokeya hapo. Kwa mfano, Baghani uko karibu na Ngome ya Fort Jesus na jina lenyewe asili yake ni la kiyarabu lenye maana ya 'vita au mapambano ya kivita', 'ujambazi' au 'uwasherati' (Al Mawrid, 1995). Katika sarufi ya Kiswahili tunajuwa kuwa kiyambishi /-ni/ ni mofimu ya kuonesha mahali. Tutaona kwamba fafanuzi mbili za mwanzo zaafiki toponimi ya mahali hapo kwa sababu ya ukuruba wake na Ngome, nripo palipopiganwa vita baina ya wananchi wakisaidiwa na Waarabu kumwonrowa Mreno. Baghaa, kwa maana ya uwasherati, maelezo haya hayakubaliyani na wazo letu kwa sababu nrani ya Mambasa kulikuwa na sehemu ikijulikana kwa uwasherati ikiitwa Msufi Mkavu karibu na Mwembe Tayari, lakini, mahali penyewe hapakuitwa kwa kazi ifanywayo hapo bali palipawa jina la $\mathrm{k}^{\mathrm{h}}$ itu maarufu kilichokuwako nrani ya $\mathrm{mt}^{\mathrm{h}}$ aa huwo, mti wa msufi, vilevile Baghani haiwezi kuchukuwa maana hiyo.

3 Toponimi ya Hisiya: ni ile inayotokana na utathmini wa hisiya ya mpaji jina mahali hapo au kuna sababu ya mlemeo mkubwa wa sifa ya hapo mahali. Mfano wa toponimi hii ni mithaa inayoitwa Kereketa (karibu na Hospitali $\mathrm{K}^{\mathrm{h}} \mathrm{uu}$ ya Mkowa,
Mambasa) na Peleleza uliyoko Likoni. Kulingana na Kindy (1972) majina ya mithaa hii ilitokana na matajiri wawili, Sir Ali bin Salim aliyeliita shamba lake Peleleza (kuchunguza wath ${ }^{\mathrm{h}}$ ) na Sheikh Rashid bin Soud Shikely akaliita shamba lake Kereketa (kukereka) kumjibu tajiri mwenziye Sir Ali kutokana na siyasa iliyokuwa ikienreleya wakati huwo kabla ya uhuru wa Kenya 1963.

4 Toponimi ya Mguro: ni ile inayoelezeya namna mahali panapopata jina lake kutokana na mahali kwengine au khitu maarufu hapo. Mfano mzuri wa aina hii ni jina Majengo au Mkunguni. Tutaona kuwa katika Mambasa kisiwani kuna Majengo aina nyingi ya Ropa (kutokana na ngoma ya Simba Ropa nriko itokako), Mferejini (palikuwa na mfereji wa kuuza maji hapo), King'orani kwenye king'ora cha kuita wat ${ }^{\text {th}}$ kazini. Ukivuka Daraja la Nyali ukifika Kisauni utakuta Majengo Mapya karibu na Msikiti Mkubwa hapo na ukienra hata Mtwapa, Kanamai kuna Majengo. Hata nrani ya Nairobi na Kisumu kuna Majengo inayokaliwa zaidi na Waswahili. Hali kadhalika kuna Mkunguni (aina ya mti) Lamu, Mambasa, na Zanzibar. Vilevile, kutokana na mti, Matonroni (Lamu) na Mtonrooni Mambasa (karibu na Kibokoni).

5 Toponimi ya Kiasili: ni ile inayoelezeya uwasili wa mahali hapo au neno lenyewe ni la kiasili. Mthaa wa Ganjoni uliyoko upanre wa Bandari ya Kilinrini nriyo sehemu asiliya ya Watangana na Wakilinrini. Kulingana na mahojiyano yangu na mwanafunzi mmoja wa Uzamifu nilipomuuliza kuhusu Kiganjo, mji uliyoko Nyeri, alinambiya Kikikuyu 'iganjo' ni 'ardhi ya wahenga' ya jamii yao. Sembuse katika Kiswahili jina hili kuwafikiyana na Watangana na Wakilinrini ambao simulizi zao zajinasibisha na huko na makaburi yao yako huko na huzikwa huko wanapofariki duniya.

6 Toponimi ya Maadhimisho: ni inayoelezeya mahali kwitwa kwa kwadhimisha au kumuenzi $\mathrm{mt}^{\mathrm{t}} \mathrm{u}$. Katika sehemu iliyoko karibu na Posta $\mathrm{K}^{\mathrm{h}} \mathrm{uu}$ ya Mambasa pana $\mathrm{mt}^{\text {haa }}$ hapo waitwa Sheikh Jundani. Pameitwa hivo kwa sababu shekhe huyu aliujenga msikiti maarufu hapo na yeye mwenyewe akazikwa hapo. Ni mahala maarufu Mambasa kwa kuzuriwa makumbusho ya shekhe huyo na wat ${ }^{\text {th }}$ kuomba baraka zake.

7 Toponimi ya maelezo: nriyo maarufu zaidi ya zote tulizozungumziya awali. Toponimi hii 
huelezeya sifa zilizoshikamana na $\mathrm{k}^{\text {hitu }}$ kilichopo katika mahali hapo. Sanasana huwa $k^{\text {hitu cha }}$ kimaumbile ambacho huipa sura sehemu hiyo kutokana nacho. Kwa mfano: mto, msitu, mlima,

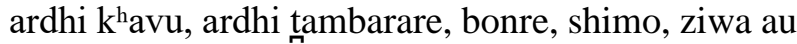
kidimbwi. Katika sehemu hii tutachanganuwa toponimi za mithaa ambayo baadhi ya fafanuzi tulizozipata nyanjani hazikubaliyani na utaratibu wa kutafiti toponimi kwa kuwa zimekengeuka na kawaida inayoonekana - toponimi za eneo lote zina sifa iliyokaribiyana ya kwita majina kutokana na maumbile. Twaeleza ifuwatavo:

\section{i) Kavani na Kibokoni}

Ni majina ya mit ${ }^{\text {thaa }}$ ambayo yapatikana katika miji mingine Kavani, Mayotte, Comoros na Kibokoni, Zanzibar. Kresse (2007) na Abdulaziz (1979) wamesema Kavani, mahali palipo na afisi au makazi ya mkuu wa serikali ya wakati wa ukoloni Wareno, uko katika $\mathrm{mt}^{\text {thaa }}$ mkubwa wa Kibokoni. Tuliyelezwa piya Kibokoni ni mahali palipoonekana/kuwako viboko (wanyama). Kresse akimnukuu Maitland-Jones amesema ni mahali palipokuwa pakichapwa wat ${ }^{\text {h }} \mathrm{u}$ na mijeledi ya ngozi ya kiboko.

Fasiri yetu ya Kavani ni kuwa katika Kimvita /w/ inaweza kuwa /v/ kwa mfano, neno /wewe/ hutamkwa /veve/ 'you'. Kwa mfanano huu basi twaweza kusema 'kavani' ni 'kawani' ambayo tukionrowa /-ni/ ya mahali itakuwa 'kawa' yenye kumaanisha chombo cha miyaa cha kufinikiya chakula; umbo lake ni kama mnara 'cone'. Kulingana na Sacleux 'viboko' ni aina ya mshono wa kanzu kama minara au mrera (mafundi wa kushona nguwo za kike walitwambiya 'mrela' au 'flare'). Kwa hivo, katika muktadha huu, kiboko si mnyama, si mjeledi ni mnara na ushahidi ni Kibokoni nriko kwenye misikiti ya minara ya kizamani, Mandhiri (piya wajulikana Msikiti Mnara) na Basheikh, na Fort Jesus yenyewe pambizo zake.

Maelezo ya pili ya Kavani inatokana na 'uvaa' kwa Kithikuu, kiwaa kwa Kimvita yenye maana ya uwanja mtupu katika eneo lenye miti mingi kama msitu wa 'savannah' (Sacleux, 1939). /ka/ katika Kavani ina maana ya udogo (Ngeli 12) na /ni/ ni ya mahali. Kwa hivo /va/ ni uwanja mtupu katika miti mingi. Nyanjani tuliyelezwa kuwa katika enzi za Wareno kulikuwa na uwanja mkubwa uliyozungushiwa ukuta katika sehemu hiyo ili kujilinra na uvamizi wa wananchi wa Mambasa ambao walikuwa wakiupinga utawala wao. Kwa hivo, Kavani ni uwanjani palipokuwa na miti mingi na majumba yaliyojengwa yalizungushiwa uwa wa mawe. Kutokana na tafsili hii twaweza kusema piya 'kavani' ni neno lengine linalomaanisha 'uwa', 'ngome', yaani hiyo Fort Jesus au ni huwo mthaa wa Wareno wenye uwa.

\section{ii) Kongoweya}

Muyaka bin Haji na Ustadh Ahmad Nassir, washairi watajika, wothe wameuwita mji wa Mambasa, $\mathrm{K}^{\mathrm{h}}$ ongoweya. Ahmad amesema nrilo lililokuwa jina la zamani kabla ya Mambasa. Katika kuuliziya kwetu wasailiwa wamesema vivo hivo na sisi piya twasema toponimi hii ni jina la kale la Mambasa. Tafauti inakuja tulipowauliza maana ya jina hili. Wothe walisema ni 'kumkaribisha $\mathrm{mt}^{\mathrm{h}} \mathrm{u}$ ' katika msemo 'mgeni kongo' yaani 'karibu mgeni'. 'K ${ }^{\mathrm{h}}$ ongoweya' kwa maana ya kumkaribisha $\mathrm{mt}^{\mathrm{t}} \mathrm{u}$ kama ilivo haikubaliyani na utaratibu wetu wa uțafiti.

Tukifuwatiliya makamusi tutrapata kuwa ' $\mathrm{k}^{\mathrm{h}}$ ongo' kulingana na Krapf (1882) ina maana ya 'mnazi ulozeeka', 'msitu ulozeeka' na Sacleux amesema ni 'tobwe', 'shimo'. Jina hili $\mathrm{K}^{\mathrm{h}}$ ongoweya twaweza kuligawanya kimofolojiya $/ \mathrm{k}^{\mathrm{h}}$ ongo- $+-\mathrm{e}-+-\mathrm{a} /$. / $\mathrm{k}^{\mathrm{h}}$ ongo-/ nriyo mzizi wa kitenzi, /-e-/ ni mofu ya kauli ya kutenreya na /-a/ ni kiishiyo. Mohamed (2001) akiyelezeya kuhusu vitenzi va kauli ya kutenreya alisema kuwa mofimu za kauli hii huweza kwelezeya hali ya kuwa - kile kitenro kiko katika kufanyika. Mohamed S. (2001: 80) ametumiya neno 'kudogoweka' kwonesha hali imo katika kufanyika kwa kauli ya kutenreya. Kwa mnasaba huu twaweza kusema kuwa $\mathrm{K}^{\mathrm{h}}$ ongoweya ni msitu mzee, wenra ukiwa mzee au ulikuwa mbichi sasa wenra ukizeekeya. /w/ na /y/ zilizomo katika maandiko ya jina hilo ni kwa sababu ya kukutana irabu thatu kwa pamoja ikabidi kwengezwe viyayusho hivo katikati yao. Kwengezeya, $\mathrm{K}^{\mathrm{h}}$ ongoweya kwa maana ya 'tobwe' 'depression' yawezekana kwa sababu ipo sehemu katika eneo hili yaitwa Ziwa la Ng'ombe, panapokusanyika maji makuu wakati wa masika.

Tafauti nyengine inayojitokeza kuhusu jina hili ni kwamba linapotamkwa huwa kuna mpumuwo /k ongoweya/ jambo aliloonesha Abdulaziz (1979) 
wakati alipokuwa akiyaandika mashairi ya Muyaka. Huu mpumuwo katika Kimvita una uwamilifu wa kutafautisha maana. Kwa mfano, tukisema /paka/ ni

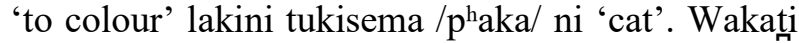
tunapotamka 'kongoweya' kwa maana ya kukaribisha hakuna mpumuwo katika /k/, hivo basi, ni maneno mawili tafauti hayo; $/ \mathrm{k}^{\mathrm{h}}$ ongoweya/ ni msitu na /kongoweya/ ni kukaribisha mtu.

\section{iii) Nyali}

Wakati wa utafiti huu nililibwaga swali la nyusuli za majina ya mji wa Mambasa kwa wanafunzi wangu na wakasema usuli wa jina hili nyali ni 'miyale(i) mikubwa iliyotokana na vita vikubwa vilivopiganwa baina ya Wareno na Waarabu'. Historiya inatwambiya vita viliyenreleya mara kwa mara mpaka 1728.

Kimofolojiya /nyali/ ni wingi wa /mwali/ ambapo Kisanifu ni /miyale(i)/ lakini kilahaja husemwa /nyali/. /mwali/ inafanana na jina la Nchi iliyoko katika Jumuiya ya Comoros inayoitwa Mohéli/Mwali [mo.e.li] piya huțamkika Moëli (Sacleux, 1939:580). Mwali piya hutamkwa 'mwalio' katika lahaja za Kusini (za Tanzania) na ina maana ya kupanga vijiti chini katika mtungi, au kuzipanga nrizi kwenye vijiti hivo (Sacleux, 1939:1014) kama wafanyavo mama zetu wanapozipika. Huku kupanga vijiti piya huitwa 'uziyo' kama wafanyavo wavuvi baharini kuwa ni mtego wa samaki. Huu 'uziyo' una mnyumbuliko wake kwa maana ya 'kiziwi' (na kuna mthaa Mambasa kwa jina hili) lenye maana hiyohiyo ya 'uziyo'. Wasailiwa wangu walinikumbusha kuwa pale forodhani mpaka Jiwe la Mbu palikuwako na nyuziyo hizi za kushika samaki. Kutokana na fafanuzi hizi yaweza kuwa Nyali $\mathrm{K}^{\mathrm{h}} \mathrm{uu}$ humaanisha nyuziyo $\mathrm{k}^{\mathrm{h}} \mathrm{uu}$ au miti mikuu na yaweza kuwa kwa kwitwa piya $\mathrm{K}^{\mathrm{h}}$ ongoweya basi palikuwa na msitu mkubwa.

Fasili nyengine tukitumiya jina la Kimijikenra 'nyari/nyali' ina maana ya ufunuwo 'cleft' (Mgombato, 2004). Kutokana na jiografiya ya ulipo $\mathrm{mt}^{\text {thaa }}$ wa Nyali leo, twaweza kusema ni ile ardhi inayokinga kisiwa cha Mambasa na miji ya nrani katika upulu huwo mpaka kufikiya ufuwo wa Rabai. Kwa mintarafu hiyo, twaweza kusema nrilo lango $\mathrm{k}^{\mathrm{h}} \mathrm{uu}$ la bahari ya kwingiliya nrani ufikiye mit ${ }^{\mathrm{h}}$ aa ya Tudor, Mikinrani mpaka Rabai kwa bahari. Hoja yetu inashikiliya kuwa ni ile ardhi inayokinga miji ya nrani kwa sababu ya umbile la lango na jina tulilopata nyanjani kutoka Mindat (2021) ni Ras Iwetini (Nyali Point) na mkabala wake ni Ras Taka, Madobini (Abdulaziz, 1979: 288).

\section{iv) Kaloleni}

Hili nrilo jina lililoleta maelezo tata kati ya majina yotre ya Mambasa. Wasailiwa woth ${ }_{n}^{\text {the }}$ kutoka wa Kiswahili mpaka wa Kimijikenra na baadhi ya wa Kipokomo walinieleza kuwa maana yake 'kaangaliyeni'. Na haswa wa Kimijikenra walinipa na historiya-simulizi ya namna mji wa Kaloleni uliyoko Wilaya ya Kilifi ulivokuja kwitwa kwa maana hii. Twaelewa kuwako na maelezo ya aina hii kwa sababu neno 'kaloleni' latokana na neno 'lola' kwa maana ya 'kuona, kwangaliya' na wingi wake ni 'kaangaliyeni' lakini utafiti wetu haukukubali fasili hii.

Nadhari yetu ilivutwa na mji katika Wilaya ya Tana River unaoitwa Galole. Mshawasha wetu ni $/ \mathrm{k} /$ katika Kiswahili huweza kugeuka na kuwa /g/ kwa mfano katika maneno /kiza/, /kețe/ huțamkwa piya /giza/ na /gete/ kimțawaliya. Nilipowauliza wasailiwa wangu wa Kipokomo kuhusu maana ya 'galole', neno siyo usuli wa mji, walinijibu kuwa kwa Kiborana na Kipokomo humaanisha 'mkono wa $\mathrm{mt}^{\mathrm{h}} \mathrm{o}$ '. Na nilipowauliza kuhusu wanauwitaje ' $m t^{\text {ho' }}$ ' wakanijibu kuwa huitwa 'chana', 'galana' 'tsana', na mchirizi 'stream' unaoingiya $\mathrm{mt}^{\mathrm{h}} \mathrm{O}$ mkubwa huitwa 'lalo' ambapo kwa Kimijikenra 'lalo' lamaanisha 'kijiji' (Mgombato, 2004). Katika fonolojiya hili laelezwa kuwa mfanyiko wa 'metathesis' (Campbell, 1998) yaani kuhamishwa sauti wakati wa kutamkwa kwake, ya mbele ikaja nyuma na nyuma ikaja mbele. Kwa mfano, katika maneno 'kibiriti//kiribiti', 'huruma/ruhuma'. $\mathrm{Na}$ tukilichukuwa kuwa latrokana na 'lola' basi lawafiki na maana ya Kiswahili kumaanisha 'kuoga' ambapo twaikuta dhana ya 'maji' ipo hapo. Kwa kushikanisha maelezo ya Kipokomo na Kiswahili kuhusu toponimi 'Kaloleni' tutaona inahusu mchirizi wa maji $\left(\mathrm{kijit}^{\mathrm{h}} \mathrm{O}\right)$. Kutokana na maelezo hayo twaweza kukatikiwa kuwa 'kaloleni' ina maana ya 'mahali kwenye $\mathrm{mt}^{\mathrm{h}} \mathrm{o}$ mdogo' hivo kukubaliyana na tasnifu ya kimaumbile.

Mtthaa ambao suala la $\mathrm{mt}^{\text {h }}$ o lapatikana katika Mambasa ni ule unaoitwa Miyuchi uliyoko katika maeneo ya Mishomoroni. Katika lugha nyengine za Kibantu ' $m t^{\text {ho' }} \mathrm{\prime}$ huitwa 'oroche' (Kikisii), 'usi' 
(Kikamba), na 'oluchi' (Kiluhya). Tutaona kuwa majina yothe matatu yanafanana na jina la $\mathrm{mt}^{\text {thaa }}$ huwo wa Mambasa, Miyuchi. Pengine siku za nyuma, au mpaka leo, kuna $\mathrm{mt}^{\mathrm{h}} \mathrm{o}$ wa msimu wa mvuwa uliyokuwa ukielekeya baharini kwa upanre huwo. Katika sehemu ya Mikinrani magharibi ya Mambasa piya pana mahali paitwa Jithoni. Niliarifiwa ni mahali padogo ambapo maji hayakupwi na ni karibu sana na Rabai; wat ${ }^{\text {th }}$ huvuka kila siku kuja kufanya kazi sehemu za Changamwe na kurudi makwao jiyoni. Neno hili 't ${ }^{\text {h}}$ oni' twalipata tena katika $\mathrm{mt}^{\text {thaa }}$ unaoitwa

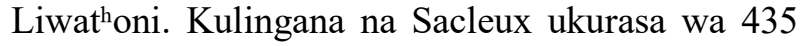
'liwa' katika Kimvita na Kiyamu ni 'mashamba' yanayolimwa nafaka na maboga. Kutokana na maelezo haya Liwat ${ }^{\mathrm{h}}$ oni /liwa $+\mathrm{t}^{\mathrm{h}}$ oni/ ni mashamba ya $\mathrm{mt}^{\mathrm{th}}$ oni (pahali pasipokosekana maji katika bahari). $\mathrm{Na} M \mathrm{t}^{\mathrm{h}}$ ongwe nayo ni majina mawili kushikanishwa $/ \mathrm{mt}^{\text {th }} \mathrm{O}+$ ngwe/. Kulingana na wa Mutiso (2005: 179) wakati alipokuwa akiyelezeya 'ngwe' katika Utendi wa Hamziya alisema ni 'kina kirefu' hivo tutaona kwamba mahali ulipo $\mathrm{mt}^{\text {thaa }}$ huu ni karibu na bahari ya Bandari ya Kilinrini ambayo humaanisha ' $m t^{\text {h }}$ o wenye kina kirefu'.

\section{v) Ingilani}

Wakați wa kuuliza kwetu kuhusu maana ya jina hili au usuli wake baadhi ya wasailiwa walitwambiya yatokana na jina 'England' (Wingereza) au 'ingiyeni'. Shauku yetu ikapatwa na neno la Kibaluhya 'ing'ani/eng'ani' lenye maana ya 'shimo' na kuna wengine walitwambiya ni 'kaburi'. Waliweza kutafautisha 'ing'ani' ambalo ni shimo niya haswa ni kufukiwa maiti na 'eshirindwa' ni shimo ambalo tayari ashafukiwa maiti. Lakini katika Kiswahili yaweza kuwa ni shimo 'depression' kama tulivosema katika $\mathrm{K}^{\mathrm{h}}$ ongoweya. Ing'ani na Ingilani yaweza kuwa ni neno moja kwa sababu /1/ imepachikwa na hili lapatikana katika Kiswahili 'kulota' na 'kuota' zothe ni 'to dream'. Na ithibati ni kwamba mandhari ya ardhi ya $\mathrm{mt}^{\text {thaa }}$ huu yațakwambiya kuwa ni shimo kwa sababu ukitoka Majestic Cinema kwenra Jamatini washuka kilima na ukitoka Barka Restaurant kuja Island Dishes piya washuka kilima na Ingilani iko chini shimoni.

\section{vi) Mvita, Mvita, Mambasa, na Kisauni}

Tumekusanya majina haya matatu pamoja kwa sababu yothe yana maelezo mamoja kuhusu nyusuli zake. Kisauni ni $\mathrm{mt}^{\text {th}}$ aa mkubwa uliyoko kaskazini ukishavuka Daraja la Nyali. Nawo Mvita/Mvita ni $\mathrm{mt}^{\text {th }}$ aa uliyoko magharibi ya kisiwa cha Mambasa na ni jina la Eneobunge. Mambasa ni jina la kisiwa chenyewe mbali ya kuwa ni jina la Wilaya. Muyaka na wasailiwa nyanjani wametaja kuwa Mvita ni kati ya majina ya kale ya Mambasa. Picha tulizoziyona katika Makumbusho ya Fort Jesus zinazo jina hili Mambasa japo limeandikwa Mombas(z)a.

Usuli tuliyoupata nyanjani umetweleza kuwa Mambasa ni 'mambo sasa', 'min bas' (kutoka kwenye shida), 'manbas'. Mvita nayo wengine wametweleza kuwa 'mji wa vita' 'mji wa nt ${ }^{\text {ha' }}$ (mji

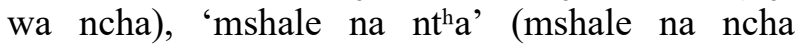
kutokana na Kikale 'mvi' ni 'mshale'). Sacleux ametaja piya 'uvi' ni mvi nyele nyeupe, mdudu nyigu, na mlango. Kisauni nayo tuliyambiwa ni 'kilifi/kisasi kisahauni', 'kisa cha wahuni'. Fasili

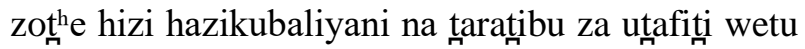
kama tunavoeleza hapa chini.

vi).1 Mvita au Mvita? Wamitila (1999) akiyangaziya mas'ala ya kifasihi anaeleza kuwa neno hili 'mvita' laweza kuwa na maana ya 'mapambano', na, akimrejeleya Sharifa Zawawi, anasema 'vita' inaweza kumaanisha 'maisha' kwa kuchukuwa maana ya Kilatini. Wazee tuliyowaomba kutweleza maana ya jina 'mvita' walilitamka /mvita/ na maelezo yao yakawa ni 'mji wa vita'. Uchanganuzi wetu kuhusu jina hili /mvita/ kutamkika vengine (ili kusikiya namna inavotamkwa angaliya video ya Hiistoriya) na kwelezwa vengine sisi tuliliyona lina mushkili.

a) Mvita ni /mvi + taa/: Katika mofolojiya iko mbinu ya kushikanisha nomino mbili kutengeza nomino moja kama ilivodhihiri kwa wasailiwa wetu. Kwa mfano, /msumeno/ inatokana na /msu/ ni upanga kikale na /meno/ 'teeth', hivo ni 'upanga wenye meno'. Hivo basi, mvita ni mshikamano wa majina mawili $/ \mathrm{mvi}+$ ta $/$.

b) /mvi/ ni /mu + vi/: Katika Kiswahili, ngeli ya binadamu, mti, na baadhi ya vitu inawakilishwa na mofimu /mu-/ au /m-/. Tunaweza kusema /mu-tu, m-tu/, /mu-ti, m-ti/, /mu-kono, m-kono/, kwa hivo, /mvi/ tunaweza kusema ni /mu-vi/. Tukichukuwa /mu-vi/ na kufuwatiliya usawe tuloeleza katika Kavani tutaona /v/ inageuka na kuwa /w/, hivo tunapata /muwi/, ambalo ni sawa na /mji/ katika Kisanifu. Kwa mintarafu hii, tutakubali maneno aloyasikiya Sacleux kwamba mvita ni 'muwi wa 
vita' kwa maana ya 'mji wa vita' japokuwa mwenyewe alikataa fasili hii (Sacleux, 1939:632) nasi ni hali kadhalika. Katika Kimvita /mu-vi/ imedonroshwa /-u-/ na kuwa /mvi/ kama tulivoeleza awali kuhusu kiyambishi cha ngeli hii.

c) Vita ni /viha/: Katika Fonolojiya sauti yenye kuleta tafauti ya maana katika maneno huwa na umuhimu wa kifonimu. Kwa mfano, tukitamka /kaa/ twamaanisha 'kuketi chini' na tukiitamka na mpumuwo $/ \mathrm{k}^{\mathrm{h}}$ aa/ ina maana ya 'mnyama wa baharini'. Vilevile, tukisema $/ \mathrm{t}^{\mathrm{h}} \mathrm{aa} / \mathrm{twamaanisha}$ 'mkoba wa kutiliya vifaa va uvuvi' na tukisema/taa/ twamaanisha 'bulb' ya kuangaziya chumba. Kwa hivo sauti /t/ ya ufizini inaleta maana nyengine na sauti /t/ ya nyuma ya meno ya juu na kwa sababu hiyo $/ \mathrm{k} /, / \mathrm{k}^{\mathrm{h}} /, / \mathrm{t} /, / \mathrm{t} \mathrm{h} /$, na $/ \mathrm{t} / \mathrm{ni}$ fonimu. Hivo basi majina haya mawili 'Mvita' na 'Mvita' kwa tafauti ya kutamkwa kwake italeta tafauti ya maana ya majina hayo na nyusuli zao.

Kwamba tafauți ya kutamka husababisha tafauti ya maana sasa tweleza maana za sauti /t/ na /t/ katika majina hayo mawili. Tukiyanza na /t/ tutaona kuwa inaafikiyana na maelezo kuwa 'mvita' ni 'mji wa vita'. Kusema hivo ni kwamba/vita/ ile /t/ ni ya ufizini na sauti hii hii twaipata kuwa ni /h/ katika Kimijikenra. Kwa mfano:

\begin{tabular}{llll} 
Kimijikenra & Kimvita & \multicolumn{2}{c}{ NKisanifu } \\
/viha/, & /vita/ & /vita/ & 'war/battle' \\
/muho/, & $/ \mathrm{mt}^{\mathrm{h}} \mathrm{O} /$ & $/ \mathrm{mto} /$ & 'river' \\
/muhi/, & $/ \mathrm{mti} /$ & $/ \mathrm{mti} /$ & 'tree' \\
/muhu/, & $/ \mathrm{mt}^{\mathrm{h}} \mathrm{u} /$, & $/ \mathrm{mtu} /$ & 'person' \\
/muhupu/ & $/ \mathrm{mtupu} /$ & $/ \mathrm{mtupu} /$ & 'empty'
\end{tabular}

Katika mifano hiyo tutaona kwamba /t/ ya Kisanifu na Kimvita inatamkwa /h/ katika maneno ya Kimijikenra. Kwa mfano /mti/ 'tree' kwa Kimvita na Kisanifu yatamkwa 'muhi' kwa Kimijikenra.

d) /ts/ ni /t/ $/$ na /s/: Tunapokuja katika /t/ ya nyuma ya meno ya juu katika Kimvita kisawe chake kinatamkwa katika maneno yaliyo na /ts/ katika Kimijikenra. Kwa mfano:

Kimijikenra Kimvita Kisanifu

\begin{tabular}{|c|c|c|c|}
\hline /mtsanga/ & /mtanga/ & /mchanga/ & 'soil/sand' \\
\hline /tsana/ & /tana/ & /chana/ & 'to comb' \\
\hline /tsaha/ & $/ t_{n}^{\text {hawa/ }}$ & /chhawa/ & 'louse' \\
\hline /mutsi/ & /muti/ & /mchi/ & 'pistle' \\
\hline /mutsi/ & $/ \mathrm{mti} /$ & /mchana/ & 'day' \\
\hline tsalafu/ & /siyafu/ & /siyafu/ & 'black ant' \\
\hline
\end{tabular}

/tsahu/ /satu/ /chatu/ 'big snake'

Katika mifano hiyo tutaona kuwa /ts/ ya Kimijikenra huwa /t/, /s/ katika Kimvita. Katika Kisanifu hugeuka na kuwa /ch/ na mara nyengine hugeuka /s/ mfano, /tsana/ husemwa /tana/ Kimvita na /chana/ 'to comb' Kisanifu. Sauti hii /ts/ ya Kimijikenra ni /t/ katika Kimvita yamesemwa piya na William Taylor (1891). Ni kweli kuwa /tsa/ katika Kimijikenra piya ina maana ya $/ \mathrm{nt}^{\mathrm{t}} \mathrm{a} / \mathrm{Kimvita}$ na /ncha/ Kisanifu kwa maana ya 'sharp point'. Lakini wasailiwa wetu jina Mvita walikuwa wakitamka /mvita/ na wala si /mvitha/ yenye mpumuwo ambayo ingeleta maana ya 'mji wenye ncha'.

e) /tsaka/, /taka/ ni msitu: Tukishaona kwamba tafauți za kutamka zinaonekana wazi sasa tuje kwenye utafautishaji maana kutokana na utamkaji ambayo nriyo shughuli ya Fonolojiya. Tukumbuke tu kuwa /mvita/ ni maneno mawili /mvi + ta/ na /t/ ya Kimvita yatamkwa /ts/ katika Kimijikenra.

Tukiregeleya Kimijikenra tutakwenraona kuwa /tsaka/ ina maana ya 'msitu'. Twangaliye neno 'msitu' kutoka lugha nyengine za karibu huitwaje:

/tsaka/ Kimijikenra

/tsaha/ Kingazija

/taka/ Kimvita

/ishaka/ Kithikuu

/itsaka/ Kitaita

/badda/ Kipokomo

/katheka/ Kikamba

/mũtitũ/ Kikikuyu 
lesichaha/ Kibaluhya

/osnet/ Kipsigis

/chaka/ Kisanifu

Katika mifano hii, tutaona /tsaka/ yenye maana ya 'msitu' sauti /ts/ inapatikana katika Kimvita, Kingazija na Kisanifu kutokana na mageuko yake kama tulivokwishakusema awali. Aidhan, ufafanuzi niliyoupata nyanjani kuhusu 'shaka' ni 'msitu mdogo wa mik ${ }^{\mathrm{h}}$ oko uliyojitenga kidogo na bara ambao umezungukwa na maji kotrt e'; ni tafauti na Kisanifu na Kitaita hurejeleya 'thicket/bush'. Tumeona kuwa maneno yotne, juu ya tafauti za utamkaji kama Kipokomo na Kipsigis, yanamaanisha 'msitu'. Kutamkwa /tsaka, tsaha, taka/ ni maana moja ya msitu kwa sababu mageuko ya /h/ kuwa /k/ na kinyumewe katika Kingazija, Kimijikenra na Kiswahili asiliya tutaona yanapatikana katika maneno /kondoo, hondoo/ 'sheep' na /konde, honde/ 'kijishamba'. Tukumbuke piya, Ras Taka (ambayo ni Madobini) imekabiliyana na Nyali na $\mathrm{K}^{\mathrm{h}}$ ongoweya ambayo tumesema yamaanisha msitu mkubwa. Sasa basi /mvi + ta/ tutaipata kwa kusema kuwa palitokeya udonroshaji wa silabi ya mwisho tukabakiwa na /ta/ katika jina /mvita/. Kutokana na uchanganuzi huu twaweza kusema, kwa hivo, /tsa/ na /ta/ ni 'msitu'. Hatimaye, /mvita/ kwa namna wanavotamka wenye lugha ya Kiswahili asiliya ni 'mji wa msitu' na wala $s i$ 'mji wa vita' kama ilivozoweleka. Utaratibu wa utafiti wetu umetanguwa dhana ya kutamka jina la mji huu vengine na kweleza usuli wake kivengine.

vi).2 Mambasa ni Mambasah? Yaonekana Mambasa yaweza kutokana na Mambasaha /mamba + tsaha/ na ukarekodiwa kama /mambasah/. Dalili ya udonroshaji wa /ha/ wapatikana katika uwandishi wa /mambasa/ kwa Kiyarabu (ممباسة) /mambasah/, yenye 'ta marbutah' ambayo hutamkwa ' $h$ ' ukikoma wakati wa kusema. Ushahidi huu uko katika pesa zilizokuwa zikitumika Afrika Mashariki 1888 B.K / 1306 B.H (Numista, 2021).

Kutokana na maelezo ya hapo juu tunaweza kusema basi Mambasa piya ni mshikamano wa majina mawili /mamba + sa/ yenye maana ya 'mwamba/mamba wa msitu'. Jamii ya Kikamba huuwita mji huu /mwambatsa/ na ya Kidawida huuwita /mambasa/ (CASAS, 2012: 12). /mwamba/ na /mamba/ ni moja kwa sababu katika Kiswahili asiliya pahali penye jiwe gumu hupaita /mwamba/ na mnyama wa $\mathrm{mt}^{\mathrm{h}}$ oni 'crocodile' humwita /mwamba/ au /mamba/ pengine kutrokana na ngozi yake ngumu. Piya Mwambasa kuwa Mambasa, uko mfanyiko huu katika lafudhi (idiolect) ya wat ${ }^{\mathrm{h}} \mathrm{u}$ kusema 'Namambiya' badala ya 'Namwambiya'. Hivo basi twaweza kusema kiasiliya Mambasa, Mambasah, Mwambatsa, Mwambasa ni alomofu za Mwambasa.

vi).3 Kisauni ni Kitaruni: Baada ya kuwona /ts/ ya Kimijikenra ni /t/ au /s/ kwa Kimvita tunaweza sasa kusema hata /kitsauni/ ni /kisauni/, /kitauni/. Kisauni inatokana na jina 'kitaruni' Kimvita kwa maana ya kijishamba kijidogo chenye kulimwa mazao machache ya kukidhi haja. Kitaru tunaweza kwigawanya kimofu /ki- + -ta- + -r- + -u/ ikawa /ki/ ni kiyambishi ngeli cha udogo (7/8), /-ta-/ mzizi wa nomino msitu kama tulivosema awali, /-r-/ kwa Kibantu ina maana ya ukubwa (kwa mfano, katika maneno /mkuru/, /shikiro/, /pura/, /guru/ ambapo tukiidonrosha /r/ maneno hayo yatakuwa /mkuu/, /shikio/, /pua/, /guu/), na /-u/ ni kiishiyo. Hivo, tumeona kuwa /ta/ imegeuka kuwa /sa/ katika Kisauni ambayo tulisema ni msitu na /ru/ ni ukubwa kwa hivo 'msitu mkubwa' (yaweza kunasibishwa na Taru mji uliyoko 80km kutoka Mambasa) lakini maana imegeuka sasa kitaru kumaanisha kijishamba kwa kwingiliwa na /ki/ ya udogo. Tukidonrosha /ni/ ambayo tumesema ni mofimu ya mahali halafu katika /kitaru/ tukidonrosha /r/ tutapata /kitau/. Tuliwauliza wasailiwa wetu kuhusu jina la sehemu ya Kitau iliyoko Wilaya ya Lamu na sifa za sehemu hiyo tuliyelezewa ni sehemu iliyoko mkabala na kisiwa cha Amu ambako nriko kwenye mashamba ya wat $^{\text {h }} \mathrm{u}$ wa Amu na Shella; na kila aliyeishi Mambasa siku za kuishi, ajuwa kwamba Kisauni mpaka leo yaitwa 'shamba' na wat ${ }^{\text {th}} \mathrm{u}$ wa 'mjini' (kisiwani).

vi).4 Mambasa au Mombasa? Swali laweza kuzuka basi ikiwa ni Mambasa/Mwambasa kefu Mombasa? Kwanza tujuwe kuwa wananchi wa Mkowa wa Pwani ambawo ni wa makamu ya miyaka 50 na kwenreleya huuwita mji huu Mambasa. Zaidi, ni Profesa Rocha Chimerah, mwalimu mzee wangu, mara zoth $_{n}^{\text {he }}$ ninazozungumza naye mpaka sasa, huutaja mji huu Mambasa, juu ya usomi wake. Hivo basi, Mombasa yaweza kuwa yatokana na masikizi ya mwandishi wa jina asiliya la Mwambasa kama ilivorekodiwa Mwali ya 
Comoro kuwa Moëli kama tulivotaja katika maelezo ya Nyali. Au, pengine walirekodi, kwa samahani (Taylor, 1891: Dibaji), lafudhi ya mtu fulani kwa sababu kunao wasemaji pwekepweke wanaosema 'monyewe' badala ya 'mwenyewe' katika lugha ya kikawaida mjini Mambasa.

Tumeona katika sehemu hii kuwa nyusuli za toponimi za mithaa ilitegemeya zaidi vitu vinavoonekana dhahiri va kimaumbile. Piya tumeona kuna tafauti ya utamkaji wa majina ya mithaa hiyo kwa Kiswahili asiliya na Kisanifu ambacho hakikuhifadhi sauti hizi katika hați yake. Tafauti hizo za kutamka jina zimetupa ufunuwo wa maana tafauti ya majina haya. Alakulihali, tumebayanisha na kuthibitisha kuwa etimolojiya ya toponimi za mithaa ya kale ya Mambasa ina miyonroko ya kutokana na mazingira na maumbile ya mahali hapo.

\section{HITIMISHO}

Lengo $\mathrm{k}^{\mathrm{h}} \mathrm{uu}$ la makala haya lilikuwa kutowa maelezo ya usuli wa toponimi ya baadhi ya mit ${ }^{\text {haa }}$ ya Mambasa. Kwa kutumiya utaratibu wa kuchunguza toponimi kietimolojiya na kutumiya Utaratibu wa Isimu Mapisi tumeweza kuchanganuwa nyusuli za majina hayo. Sababu ni kwamba dhana iliyojidhihirisha wakati wa utafiti wetu tulikuta wasailiwa wana maoni tafauti tafauti kuhusu asili ya majina ya mit ${ }^{\text {haa }}$ hii. Tulichaguwa wazee wa miyaka 50 na zaidi ili kupata data ya kimsingi kuhusu utamkaji na fasili za maana ya miji hiyo. Tuliyona sampuli ya wasailiwa hawa nriyo stahikifu kwa kucheleya kwathiriwa na Kisanifu ambacho hakikuhifadhi sauti $\mathrm{zoth}_{n}^{\text {the }}$ za Kiswahili asiliya. Japo mas'ala ya kiisimu-mapisi yana kiwango cha udhahanifu lakini tuliweza kupunguza athari hii kwa kurejeleya maandiko yaliyoko yenye maelezo ya toponimi hizi.

Kutrokana na Utaratibu wa Ulinganisho wa visawe kutoka lugha kadhaa zinazokurubiyana na Kiswahili: Kimijikenra, Kipokomo, Kingazija, na kwa uchache lugha za Kibantu za bara ya Kenya tumeweza kubainisha kuwa, mwanzo, lugha hizi zina uhusiyano katika ngazi za kifonolojiya, kimofolojiya, na kisemantiki. Pili, athari za kisemantiki piya zimedhihirika katika lugha tulizochanganuliya data yetu na hivo kusakinisha madhumuni yetu kwamba maana za toponimi za mithaa ya kale ya Mambasa zatokana na maumbile ya mazingira sawiya katika lugha nyengine za Kibantu: Ganjoni, Kaloleni kutoka Mambasa, na Kiganjo, Galole, huko Nyeri, Tana River kimtawaliya. Aidhan, tumefunukiwa kwamba tafauti za kimatamshi ya sauti na mageuko yake katika matumizi huleta tafauti za nyusuli za majina ya mithaa ya kale ya Mambasa, kwa mfano, Mvita, $\mathrm{K}^{\mathrm{h}}$ ongoweya, Mwambasa; sauti $/ \mathrm{t} / \mathrm{ya}$ Kiswahili asiliya imetafautisha usuli na maana ya jina la mji huu. Tumefunukiwa piya katika utafiti huu kwamba mithaa ya hivi karibuni imepata toponimi zake kutrokana na mazingira ya kukurubiyana na jengo (Ngomeni), matukiyo (Baghani), maenreleo mapya katika mji (Kavani), majenzi ya nyumba kimpangiliyo (Majengo), au, ni hisiya za mpaji jina (Kereketa). Makala haya yameleta wazo jipya kuhusu kujenga dhana katika mas'ala ya kitamaduni, kimapisi, kiisimu, na chimbo za mji wa Mambasa. Kuhusu wazo hili twaweza kusema nini kuhusu toponimi za miji mengine katika Mkowa wa Pwani au Kenya nzima kwa faida ya mapisi ya jamii zetu? Je, utafiti wetu umefunuwa milango ipi ya

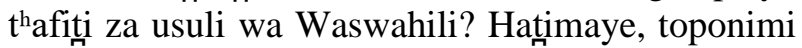
hizi zawezaje kugeuzwa kuwa bidhaa za kiutalii ili zivutiye watalii kutembeleya miji hii?

\section{SHUKURANI}

Nawashukuru ghaya wat $^{\mathrm{h}} \mathrm{u}$ woth ${ }^{\mathrm{th}} \mathrm{e}$ walonisaidiya wakati wa matayarisho ya makala haya wakiwamo Prof. Rocha Chimerah, PU; Prof. Issa Mwamzandi, UoK; Dkt. Vifu Makoti, MKSU; Dkt. Hamisi Babusa, KU; Dkt. Kalandar Kamalkhan, TUM; Athman Hussein, Mohamed Mwenje, Khalid Kitito, Saadu Hashim, na Mbarak Abdulkadir wote wa NMK; Washairi Hassan Morowa na Abdilatif

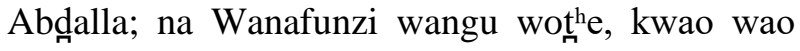
nawatnunukiya makala haya.

\section{MAREJELEO}

Abdulaziz, M. H. (1979). Muyaka: 19th century Swahili popular poetry. Kenya Literature Bureau.

Antilla, R. (1989). Historical and comparative linguistics. John Benjamins Publishing Company. 
Bynon, T. (1977). Historical linguistics. Cambridge University Press.

Campbell, L. (1998). Historical linguistics: An introduction. Edingburgh University Press.

Centre for Advanced Studies of African Society, (2012). A unified orthography for Bantu languages of Kenya. Monograph no. 249.

Helander, K. R. (h.t.). Renaming indigenous toponymy in official use in light of contact onomastic theories. Sami University College, Norway.

Hiistoriya, (2020, Jul 12). Who are the Swahili? [video file]. Imetolewa https://www.youtube.com/watch?v=pghUezn9F E4

Karama, M. R. (2021, June). Kugeuza dira ya toponimi ya 'Swahili'. The University of Kabianga Newsletter 1(5), 8-9.

Kindy, H. (1972). Life and politics in Mombasa. East African Publishing House.

Krapf, L. (1882). A dictionary of Suahili language. Trubner \& Co.

Kresse, K. (2007). Philosophising in Mombasa: Knowledge, Islam and intellectual practice on the Swahili coast. Edinburgh University Press.

Makoti, V. S. (2007). Digo anthroponymes: Meaning and significance. Maarifa: A Journal of Humanities and Social Sciences 2 (1) 18-28.

Mazrui, A. A. (2012). What's in a name?: European imperialism and the re-naming of Africa. Kandi ya A. Mazrui, State University of New York at Binghamton, New York.

Mindat.org. (2021, June). Ras Iwetine, Mombasa, Kenya. Ilisomwa kutoka https://www.mindat.or g/feature-196036.html .

Mohamed, M. A. (2001). Modern Swahili grammar. East African Educational Publishers.

Mohamed, S. A. (2001). Babu alipofufuka. Jomo Kenyatta Foundation. wa Mutiso, K. (2005). Utenzi wa Hamziyya. TUKI.

Mutua, M. (2021, June 20) It's time to decolonise Kinyaa. Nation.Africa. Ilisomwa kutoka https://nation.africa/kenya/blogsopinion/opinion/it-s-time-to-decolonise-kinyaa3443516

Mwalonya, J., Nicolle, A., Nicolle, S. \& Zimbu, J. (2004) Mgombato: Digo-English- Swahili dictionary. Bible Translation Literacy.

Numista, (2021, June 2). IBEA coinage.

Ilisomwa kutoka https://en.numista.com/catalogue /pieces8981.html

Nurse, D. \& Spear, T. (1985). The Swahili: Reconstructing the history and language of an African society 800-1500. University of Pennsylvania Press.

Nzasu, J. \& Odongo, W. (2014). Our county: Mombasa. Oxford University Press.

Owino, S. (2019, October 27). What's in a name? Take heed in these legal views, implications. Kenyan Digest. https://kenyandigest.com/whats-in-a-nametake-heed-in-these-legal-views-implications/

Rose-Redwood, R., Alderman, D. \& Azaryahu, M. (2010). Geographies of toponymic inscription: New directions in critical place-names studies. Progress in Human Geography 34(4), 453-470.

Sacleux, C. S. (1939). Dictionnaire SwahiliFrancais. Institut d' Ethnologie.

Stigand, C. H. (1915). A grammar of dialectic changes in the Kiswahili language. Cambridge University Press.

Taylor, W. E. (1891). African aphorisms. Society for Promoting Christian Knowledge.

Tent, J. \& Slatyer, H. (2009). Naming places on the Southland: European place-naming practices from 1606 to 1803. Australian Historical Studies 40(1), 5-31.

Tent, J. (2015). Approaches to research in toponymy. $\quad$ Names 63(2), 65-

89 | This work is licensed under a Creative Commons Attribution 4.0 International License. 
74. https://doi.org/10.1179/0027773814z.00000 0000103 .

Walsh, M. T. (2003). Hunter-gatherers in the hinterland of Mombasa: Notes on the Maumba of Chonyi and related traditions. Notes.

Wamitila, K. W. (1999). What's in a name: Towards literary onomastics in Kiswahili literature. AAP 60, 35-44.

Wanjiru, M. W. \& Matsubara, K. (2017). Street naming and the decolonisation of the urban landscape in post-colonial Nairobi. Journal of Cultural Geography 34(1), 1-23.

https://doi.org/10.1080/08873631.2016.120351

8.

90 This work is licensed under a Creative Commons Attribution 4.0 International License. 